\title{
Avaliação de Potenciais Fragmentados em Presença de Bloqueio de Ramo Direito sem Alterações Estruturais Miocárdicas Ventriculares. Estudo pelo Eletrocardiograma de Alta Resolução no Domínio da Freqüência
}

\author{
Paulo Ginefra, Eduardo C. Barbosa, Luiz A. Christiani, Maria de Fátima M. P. Leite, \\ Paulo R. Barbosa, Sílvia H. Boghossian, Mônica Scott, Isabela M. T. Rangel, \\ Rosângela S. Silva, Francisco M. Albanesi Fo
}

Rio de Janeiro, RJ

\begin{abstract}
Objetivo - Em ausência de alterações estruturais miocárdicas (AEM), avaliar se o bloqueio de ramo direito (BRD) gera potenciais fragmentados $(P F)$ e turbulência espectral (TE) no eletrocardiograma de alta resolução (ECGAR).

Métodos - Doze crianças com comunicação interatrial (CIA) e bloqueio incompleto do ramo direito (BIRD) sem AEM (Grupo I), foram comparadas com 17 crianças com tetralogia de Fallot (TF) operada, BCRD e AEM, 5 com extra sístoles ventriculares e $2 \mathrm{com}$ taquicardia ventricular sustentada (Grupo II). Todas fizeram ECGAR nos domínios do tempo (DT) e da freqüência $(D F)$, com cinco variáveis analisadas.

Resultados - Os pacientes do grupo I tiveram as variáveis normais apesar do BIRD. No grupo II, 4 das cinco variáveis foram anormais, sugerindo a presença de $P F$ e TE atribuíveis a AEM inerentes à malformação e ao ato cirúrgico.

Conclusão - Na CIA o BIRD não complicado de AEM não gera PF e TE, não constituindo fator de risco para taquicardia ventricular sustentada.
\end{abstract}

Palavras-chave: eletrocardiograma de alta resolução, turbulência espectral, bloqueio do ramo direito.

\section{Evaluation of Fragmented Potentials in the Presence of Right Bundle Branch Block without Structural Myocardium Abnormalities by Using Signal-Averaged Electrocardiogram on Time and Frequency Domain}

Purpose-To evaluate if the presence of right bundle branch block $(R B B B)$, without structural myocardial abnormalities (SMA) can generate fragmented potentials (FP) and spectral turbulence on signal-averaged electrocardiogram (SAECG).

Methods - Twelve children with atrial septal defect (ASD) and incomplete right bundle branch block (IRBBB without SMA (group I) were compared to 17 children with post-operative tetralogy of Fallot (TF) with $C R B B B$, all with SMA, 5 with ventricular premature beats and 2 with sustained ventricular tachycardia (group II). All had SAECG on time (TD) and frequency domain (FD) with 5 variables analysed.

Results - All patients of group I had normal variables, in contrast with group II which presented abnormal variables suggesting FP and ST.

Conclusion -In ASD without SMA, the isolated IRBBB did not generate FP and ST.

Key-words: signal-averaged electrocardiogram, spectral turbulence, right bundle branch block.

Arq Bras Cardiol, volume $71\left(n^{0} 5\right), 687-694,1998$

Faculdade de Ciências Médicas da Universidade do Estado do Rio de Janeir Correspondência: Paulo Ginefra - UERJ - Av. 28 de Setembro, 77 - $2^{\circ}$ - 20551-030 - Rio de Janeiro, RJ

Recebido para publicação em 18/5/98 Aceito em 14/8/98
O miocárdio normal é constituído de miofibrilas que se organizam em feixes de fibras musculares, que se dispõem em paralelo, formando grupos de tecido miocárdico orientados para constituir várias camadas musculares que, por sua 
vez, vão compor as paredes cardíacas. A essa orientação das fibras miocárdicas em paralelo, Spach e col ${ }^{1-3}$ denominaram de estrutura miocárdica anisotrópica uniforme e sua maneira de se organizar, destina-se a permitir a propagação do estímulo elétrico paralela à orientação das fibras ao longo de seu eixo longitudinal, de modo mais rápido, tanto nos átrios como nos ventrículos.

Uma lesão ou escara desarranja essa organização, caracterizando a chamada estrutura anisotrópica não-uniforme, ou seja, uma alteração estrutural miocárdica (AEM) em que a condução do estímulo elétrico não se faria preferencialmente no sentido longitudinal das fibras, mas sim, em sentido perpendicular entre elas, ocasionando o alentecimento da condução elétrica na área lesada, devido à redução da conexão intercelular ${ }^{2}$, alargando e fracionando o potencial elétrico dessas fibras, constituindo os chamados potenciais fragmentados ${ }^{4} \mathrm{em}$ nível celular, de alta freqüência e de muito baixa voltagem.

Estes eventos ocorrem gradualmente durante a evolução dos infartos do miocárdio, processos esclero-degenerativos, degeneração gordurosa, seqüelas de algumas miocardites, áreas de fibrose intercalada, que geram instabilidade elétrica com dispersão de períodos refratários por repolarização heterogênea entre fibras, constituindo o substrato de mecanismos de micro-reentradas ${ }^{2-4}$.

O eletrocardiograma de alta resolução (ECGAR) constitui o único método não-invasivo capaz de detectar potenciais fragmentados da ordem de microvolts, oriundos daquelas áreas estruturalmente alteradas, e que podem ser registrados com metodologias no domínio do tempo(DT) e no domínio da frequiência (DF) ${ }^{5-7}$. No primeiro, analisam-se três variáveis, sendo duas em função da voltagem e duração dos eventos de interesse nos 40ms finais do complexo QRS, e uma variável relacionada ao tempo total da ativação ventricular, que corresponde à duração total do QRS (DQRS). No DFe a partir das medidas do método anterior, avaliam-se quatro índices em função das ondas de frequiência em Hertz geradas pelos potenciais fragmentados, mediante técnicas de correlação espectral e análise espectro-temporal, ondas essas que podem ocorrer em qualquer segmento do QRS, e não somente nos $40 \mathrm{~ms}$ finais ${ }^{7,8-10}$. A análise espectro-temporal tem maior sensibilidade e especificidade que o método do DT e maior acurácia para identificar pacientes suscetíveis de desenvolverem taquicardia ventricular sustentada ${ }^{9-12}$.

O ECGAR no DT tem limitações em presença de bloqueios de ramo, já que estes aumentam o tempo de ativação ventricular, alargando o QRS e aumentando sua duração total. Como as outras duas variáveis analisam-se nos $40 \mathrm{~ms}$ finais, com o alargamento do QRS, são abrangidas pela duração total do QRS e invalidam-se ${ }^{7,8}$. No DF, entretanto, a análise espectro-temporal permite avaliar a presença de potenciais fragmentados pelas ondas de frequiência, sob a forma do QRS alargado pelo bloqueio de ramo, sendo este método o ideal para detecção desses potenciais em meio a distúrbios da condução intraventricular ${ }^{9,13-15}$ Sendo o bloqueio de ramo um evento que retarda o processo de ativação ventricular, teoricamente é possível admitir-se que a área onde ocorre o bloqueio, ela mesma, possa gerar turbulência elétrica, não propriamente por presença de AEM com potenciais fragmentados, mas pelos retardos sofridos pela onda de ativação com deslocamento por vias alternativas.

Com a finalidade de validar esta hipótese, registrou-se o ECGAR nos DT e DF em crianças portadoras de comunicação interatrial (CIA) com bloqueio incompleto do ramo direito(BIRD), sem AEM ventriculares, que foram comparadas com outro grupo de crianças com tetralogia de Fallot (TF) operadas, todas com bloqueio completo de ramo direito (BCRD) e AEM decorrentes do ato cirúrgico e de outros fatores inerentes a esta malformação ${ }^{16}$.

O objetivo do presente trabalho é verificar pelo método do DF, se o bloqueio de ramo não complicado com AEM, como no caso da CIA, é capaz de gerar turbulência elétrica, como observado no bloqueio de ramo complicado com AEM no pós-operatório da TF, e avaliar suas implicações com eventos arrítmicos ventriculares

\section{Métodos}

Foram estudadas, com o consentimento dos pais, 29 crianças, 12 portadoras de CIA tipo ostium secundum, sendo um caso associado a canal arterial persistente, sete do sexo feminino, de idade média 5,4 $\pm 3,3$ anos, todas com BIRD no eletrocardiograma (ECG) convencional, não submetidos a correção cirúrgica (grupo I), comparadas com 17 crianças com TF corrigida cirurgicamente, sendo nove do sexo feminino, com idade média de 9,5 \pm 3 anos (grupo II).

Todos os pacientes foram avaliados clinicamente e os diagnósticos estiveram baseados em exames radiológicos, eletrocardiográficos, ecocardiográficos e, em alguns casos, estudo hemodinâmico.

No grupo I investigaram-se outras malformações associadas e antecedentes de enfermidades ou infecções prévias, que pudessem deixar seqüelas miocárdicas ventriculares, arritmias cardíacas de qualquer tipo e correções cirúrgicas, constituindo estes os principais critérios de exclusão de pacientes, baseados em dados de ECG, ecocardiográficos e estudo hemodinâmico.

O grupo II, constituído de 17 pacientes com diagnóstico de TF, operados para correção, que foi realizada através da abertura do trato de saída do ventrículo direito (VD) na região infundibular. Foi feita ressecção de extensão parietal do septo interventricular (SIV) para aumentar o diâmetro do infundíbulo e a comunicação interventricular (CIV) foi fechada e o anel pulmonar ampliado. No pós-operatório imediato, todos os pacientes já apresentavam BCRD, sendo que em dois $(11,7 \%)$ associado a bloqueio divisional ântero-superior do ramo esquerdo, todos conseqüentes ao ato cirúrgico. Durante o seguimento pós-operatório, persistiram sinais de hipertrofia ventricular direita e cinco $(29,4 \%)$ pacientes apresentaram extra-sístoles ventriculares freqüentes e dois $(11,7 \%)$ desenvolveram surtos de taquicardia ventricular monomórfica sustentada, mas nenhum de nossos casos teve morte súbita, até a elaboração da presente comunicação. Os dados clínicos, eletrocardiográficos, 
ecocardiográficos e do ECGAR e a presença dos eventos arrítmicos sugeriram-nos a existência de AEM em todos os casos deste grupo ${ }^{16}$.

O estudo radiológico auxiliou na avaliação do crescimento do VD, e nos aspectos da artéria pulmonar e hilos pulmonares. O ecocardiograma e o Doppler foram empregados para a avaliação das dimensões das cavidades e espessura e movimento das paredes cardíacas, fluxos e cálculos das pressões e da configuração da malformação.

OECG foi o convencional de 12 derivações em que foram determinados o eixo elétrico ÂQRS, a configuração do QRS em V e sua duração, a amplitude da R' de $V_{1}$, os aspectos do segmento $\mathrm{S}$-T nesta derivação e presença de $\mathrm{s}$ em $\mathrm{V}_{6}$.

Adotamos como critério de normalidade a duração total de QRS (dQRS) de 0,06s $\pm 0,01$ em média ${ }^{17,18}$, e de BIRDe BCRD a duração de QRS entre 0,08 e 0,10 s e >0,10s, respectivamente, em $\mathrm{V}_{1}$ na faixa etária de cinco e seis anos de idade ${ }^{18}$, a mesma de nossos paciente com CIA (grupo I).

OECGAR foi realizado com equipamento ART(Texas) Corazonix modelo Predictor IIc, empregando-se as três derivações ortogonais $\mathrm{X}, \mathrm{Y}$ e Z de Frank, sendo promediados $500 \mathrm{bpm}$ aproximadamente, com freqüência de amostragem de 40 a $200 \mathrm{~Hz}$, até a obtenção da redução final do ruído para $0,3 \mu \mathrm{V}$. Os registros foram realizados nos DT e DF, conforme descrito adiante. No primeiro, empregou-se um filtro bidirecional de Butterworth de quatro pólos, com cortes de 40 a $250 \mathrm{~Hz}$, convertendo-se as três derivações em um vetor magnitude resultante dos valores médios de cada derivação. Estudaram-se a DQRS (duração do complexo QRS em $\mathrm{ms}$ ), $\mathrm{RMS}_{40}$ (40ms finais do QRS filtrado em microvolts $\mu \mathrm{V}$ ) e $\mathrm{LAS}_{40}$ (duração total dos sinais de baixa amplitude e alta freqüência dos 40ms finais do QRS), considerando-se como valores normais na faixa etária em estudo, a DQRS $=73,3 \mathrm{~ms}$, a RMS $=215,8 \mu \mathrm{V}$ e a LAS $=15,3 \mathrm{~ms}^{16}$.

No DF empregou-se a metodologia preconizada por outros autores ${ }^{12}$ e já descrita em trabalho anterior em nosso Serviço ${ }^{19}$ que consiste em se definir uma área que preceda o QRS em $25 \mathrm{~ms}$ e que dure $200 \mathrm{~ms}$ com o ponto inicial obtido do vetor de velocidade espacial, fornecido pelo algoritmo do sistema do Predictor IIc. O mapa espectral tempo-freqüência é construído, dividindo-se a área de análise em segmentos de $25 \mathrm{~ms}$ de duração, multiplicados a uma janela de Blackman-Harris, separados a intervalos de $2 \mathrm{~ms}$ e aplicando-se a transformada de Fourier com 64 pontos de ordenação a cada um daqueles segmentos, para se determinar o conteúdo de freqüência do sinal ${ }^{12,19}$. Para a análise espectral, o programa emprega quatro índices: a média (Cem) e o desvio-padrão (Cedp) da correlação espectral (CE) intersegmentar e a média (Bdm) e o desvio-padrão (Bddp) da banda de freqüência (BD) delimitadora (edge track) da concentração de energia do sinal elétrico ${ }^{19}$.

A CE analisa a distribuição da amplitude do espectro de potência do sinal em função da freqüência em que ele ocorre, estabelecendo uma correlação linear de Pearson entre segmentos consecutivos de $2 \mathrm{~ms}$ ao longo da ativação ventricular (o $1^{\circ} \operatorname{com~o~} 2^{\circ}$, o $2^{\circ}$ com o $3^{\circ}$ e assim sucessivamente). De todas as correlações realizadas, são determinadas a Cem e o Cedp.
A BD identifica, para cada segmento analisado, qual a frequiência que delimita a concentração do sinal elétrico em $80 \%$ abaixo e $20 \%$ acima desta freqüência. De todos os segmentos analisados, também são determinados a média (Bdm) e o desvio-padrão (Bddp) em Hertz ${ }^{19}$.

A análise espectral no DF é realizado no somatório das três derivações x, y e z. O programa estabelece que, quanto mais graves os distúrbios da condução intramural ventricular, maiores serão os índices de correlação espectral Cedp, Bdme Bddp e menor o de Cem. ${ }^{19}$

É feita, também, a análise continuada dos dois domínios, considerando-se positivo o ECGAR para existência de potenciais fragmentados, ou tardios quando pelo menos duas variáveis do DT e dois índices do DF estejam alterados em relação aos mesmos em indivíduos normais ${ }^{16}$.

Os valores dos índices de correlação espectral em crianças normais na faixa etária dos casos aqui estudados são: $\mathrm{Cem}=96 \pm 1 ; \operatorname{Cedp}=5 \pm 1 ; \mathrm{Bdm}=64,7$ e Bddp $=24 \pm 36$, aceitos como referência ${ }^{16}$ e fornecidos em decimais pelo programa, porém multiplicados por 100 para simplificar os resultados. Valores maiores que os de referência dos três últimos índices e menores que os do primeiro índice, são considerados como indicadores da presença de potenciais fragmentados, mesmo em presença de bloqueio de ramo ${ }^{12}$.

\section{Resultados}

Estudo radiológico - Todos os pacientes do grupo I tinham o VD aumentado nas radiografias de tórax e a artéria pulmonar e hilos pulmonares apresentavam-se com características compatíveis com o diagnóstico de CIA. No grupo II, as radiografias revelaram fluxo pulmonar normale, naqueles pacientes sem complicações pós-operatórias, a área cardíaca permaneceu dentro dos limites da normalidade e pôde-se notar o aparecimento da silhueta do arco pulmonar $\left(2^{\circ}\right.$ arco $)$, antes ausente.

Na presença de complicações pós-operatórias, a imagem radiológica era variável. As principais complicações foram a presença de CIV residual, com imagem de aumento de cavidades esquerdas e hiperfluxo pulmonar, quando o defeito residual era hemodinamicamente significativo e a presença de abaulamento do arco pulmonar, com alguns casos de aumento e algumas vezes aneurisma do VD.

Ecocardiograma - No grupo I, a dimensão média do VD foi de $1,73 \mathrm{~cm}$, considerado ligeiramente aumentado para a faixa etária em estudo, com a pressão em nível da artéria pulmonar calculada em torno de $34,5 \mathrm{mmHg}$. (valor médio). No grupo II, as alterações pós-operatórias foram variáveis. Nos casos sem complicações pós-operatórias, a via de saída do VD (VSVD) mostrou-se ampliada com diminuição ou ausência de gradiente em relação ao pré-operatório e diagnosticou-se fechamento da CIV.

As complicações mais comuns foram presença de obstrução residual pela VSVD, estenose residual dos ramos pulmonares, regurgitação pulmonar com dilatação aneurismática da VSVD e presença de CIV residual.

Eletrocardiograma - Entre os grupos I e II, os resultados encontram-se na tabela I: nos grupos I e II, todos os 


\begin{tabular}{|c|c|c|c|c|c|c|c|c|}
\hline \multirow[b]{2}{*}{$\begin{array}{l}\text { Grupo I } \\
\text { CIA }\end{array}$} & $\begin{array}{l}\text { ÂQRS } \\
0^{\circ}\end{array}$ & & $\begin{array}{l}\text { Aspecto } \\
\text { predominante } \\
\text { QRS V }_{1} \\
\%\end{array}$ & \multicolumn{2}{|l|}{$\begin{array}{l}\text { Voltagem } \\
\mathrm{m} \\
\mathrm{R}^{\prime} \mathrm{V}_{1} \\
\mathrm{Mv}\end{array}$} & \multicolumn{2}{|l|}{$\begin{array}{l}\text { Duração } \\
\text { QRS } \\
\text { (dQRS) } \\
\mathrm{s}\end{array}$} & $\begin{array}{l}\text { Segmento } \\
\text { S-T } \\
\%\end{array}$ \\
\hline & $+120^{\circ}$ & $\begin{array}{l}112,37 \\
127,63\end{array}$ & rs R' $(66,6)$ & 0,91 & $\begin{array}{l}0,69 \\
1,13\end{array}$ & 0,08 & $\begin{array}{l}0,07 \\
0,09\end{array}$ & $\begin{array}{l}\text { Negativo } \\
\text { Assimetr. } \\
100\end{array}$ \\
\hline $\begin{array}{l}\text { Grupo II } \\
\text { Tetralogia } \\
\text { allot operado }\end{array}$ & $+168^{\circ}$ & $\begin{array}{l}151,97 \\
184,03\end{array}$ & rs R' $(70,5)$ & 1,9 & $\begin{array}{l}1,62 \\
2,18\end{array}$ & 0,14 & $\begin{array}{l}0,13 \\
0,15\end{array}$ & $\begin{array}{l}\text { Negativo } \\
\text { Assimetr. }\end{array}$ \\
\hline & $\mathrm{p}=<0, \mathrm{c}$ & & & $\mathrm{p}=<0,01$ & & $\mathrm{p}=<0,01$ & & \\
\hline
\end{tabular}

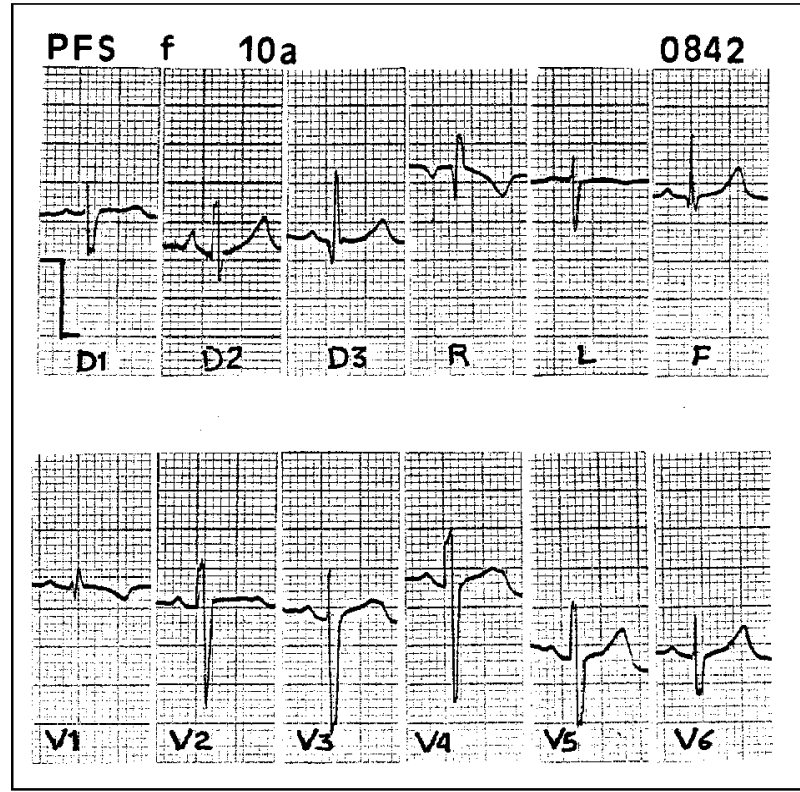

Fig. 1 - Eletrocardiograma do caso 10 do grupo I (CIA), menina de 10 anos de idade. ÂQRS a $+100^{\circ}, \mathrm{QRS}=0,08$ s de duração, configuração de bloqueio incompleto do ramo direito.

pacientes estavam em ritmo sinusal com intervalo P-R dentro dos limites da normalidade. No grupo I, o eixo elétrico ÂQRS situou-se $\mathrm{a}+120^{\circ}$ (intervalo de confiança (ic) $112,37 \mathrm{a}$ 127,63), a morfologia predominante do $\mathrm{QRS}$ em $\mathrm{V}_{1}$, foi tipo rsR' em $66 \%$ dos casos comaR' de $0,91 \mathrm{mv}$ (ic $=0,69$ a 1,13 ) de amplitude; a duração do QRS (dQRS) foi de $0,08 \mathrm{~s}$ (ic $=0,07$ a $0,09)$ com presença de onda $\mathrm{em}_{6}$ e o segmento $S$ - $\mathrm{T}$ negativo assimétrico em $\mathrm{V}_{1}$ em todos os casos (tab. I, fig. 1). No grupo II, o ÂQRS situou-se a $+168^{\circ}$ (ic $=151,97$ a 184,03), a morfologia de maior incidência em $V_{1}$ foi rsR' em $12(70,5 \%)$ casos, seguida do tipo rR' em quatro $(23,5 \%)$ casos, com a R' de $1,9 \mathrm{mv}$ e a duração média de $\mathrm{QRS}$ de $0,14 \mathrm{~s}$ (ic $=0,13$ a 0,15), ondas s espessas em $\mathrm{V}_{6} \mathrm{eS}$-T negativo assimétrico em todos os casos (tab. I, fig. 2). Neste grupo, cinco $(29,4 \%)$ pacientes tinham extra-sístoles ventriculares freqüentes e dois, episódios de taquicardia ventricular monomórfica sustentada. Comparando-se os valores eletrocardiográficos entre os dois grupos, observou-se que o ÂRQS esteve mais orientado para a direita no grupo II que no grupo I $\left(168^{\circ} \mathrm{vs}\right.$

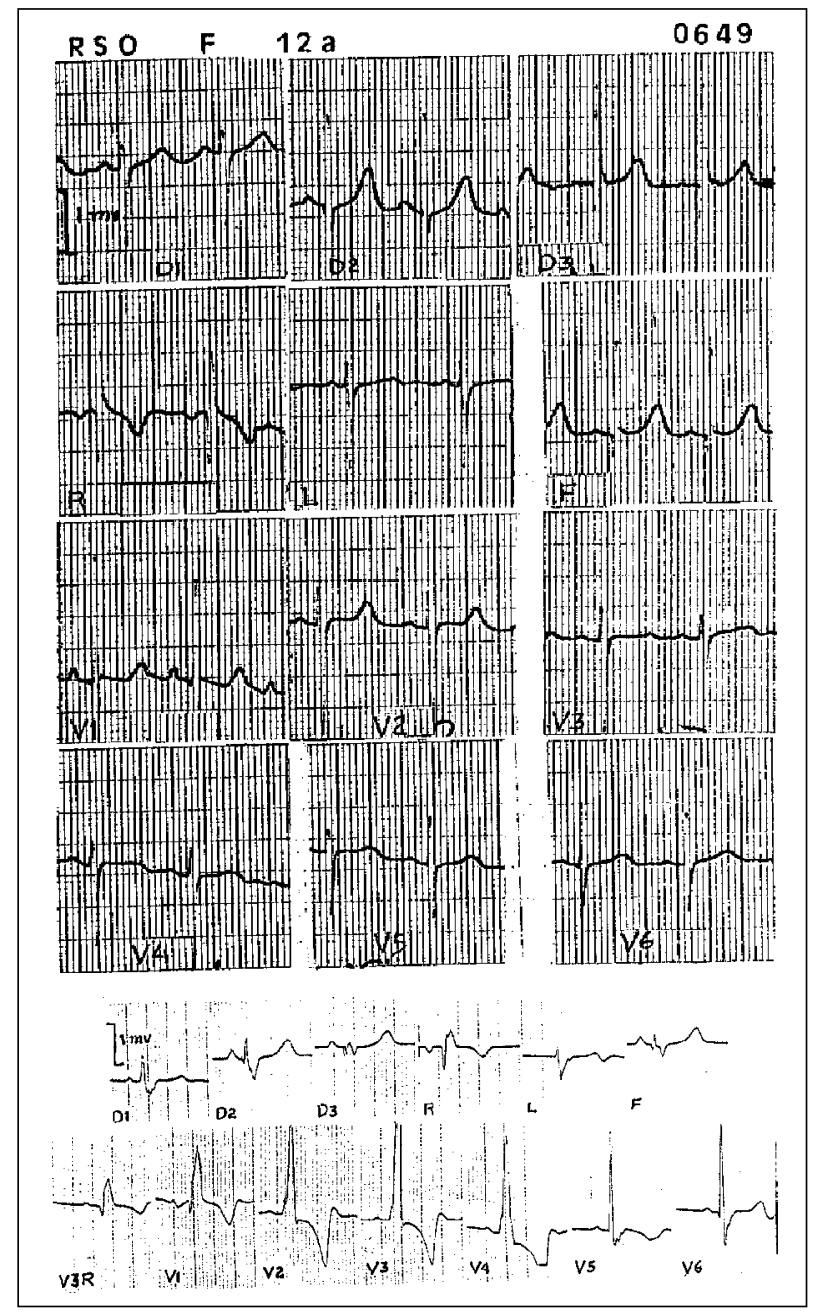

Fig. 2 - Eletrocardiogramas do caso 3 do grupo II (tetralogia de Fallot), menina de 12 anos de idade. Acima, o ECG pré-operatório, $\mathrm{AQRS}$ a $+150^{\circ}, \mathrm{QRS}=0,07 \mathrm{~s}$ de duração, aspecto de hipertrofia ventricular direita. Abaixo, ECG pós-operatório, ÂQRS em torno de $-160^{\circ}$, QRS $=0,14$ s de duração com $\mathrm{R}$ de alta voltagem de $\mathrm{V}_{1} \mathrm{aV}_{4}$, aspectos de bloqueio completo do ramo direito com sobrecarga dessa câmara.

$120^{\circ},(\mathrm{p}<0,01)$, a R' de $\mathrm{V}_{1}$ era mais ampla no grupo II $(1,9 \mathrm{mv}$, vs $0,91 \mathrm{mv}(\mathrm{p}<0,01)$ e a duração do QRS (dQRS) também foi maior no grupo II $(0,13 \mathrm{~s}$ vs 0,08 , p $<0,01)$ (tab. I). Estas diferenças foram devidas, provavelmente, à hipertrofia ventricular direita do grupo II, que persistiu com o espessamento 


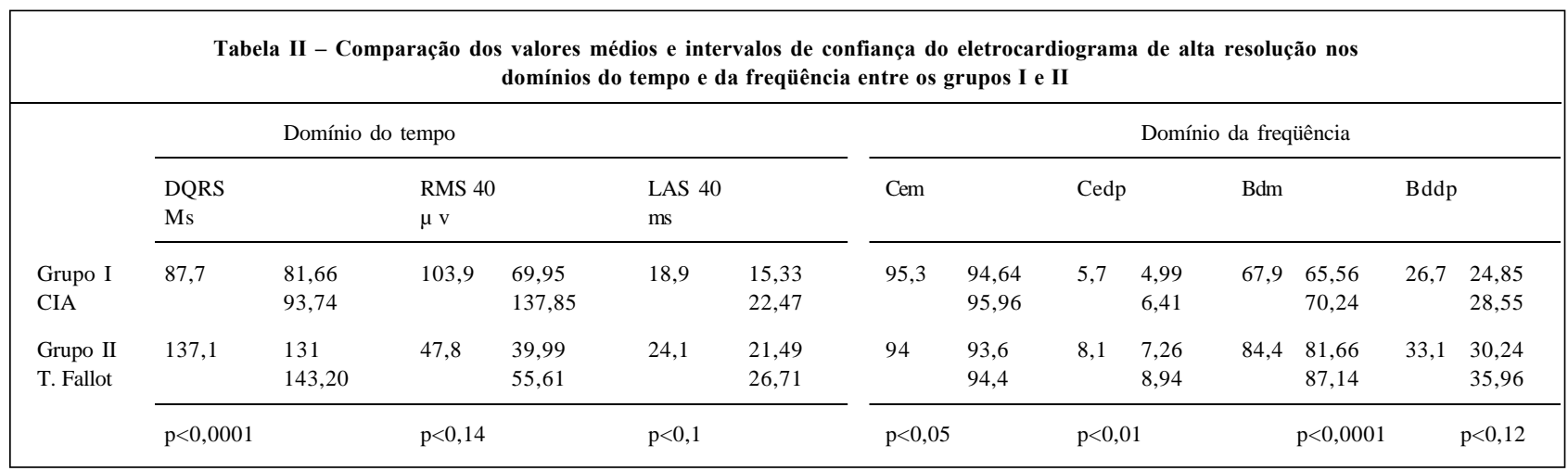

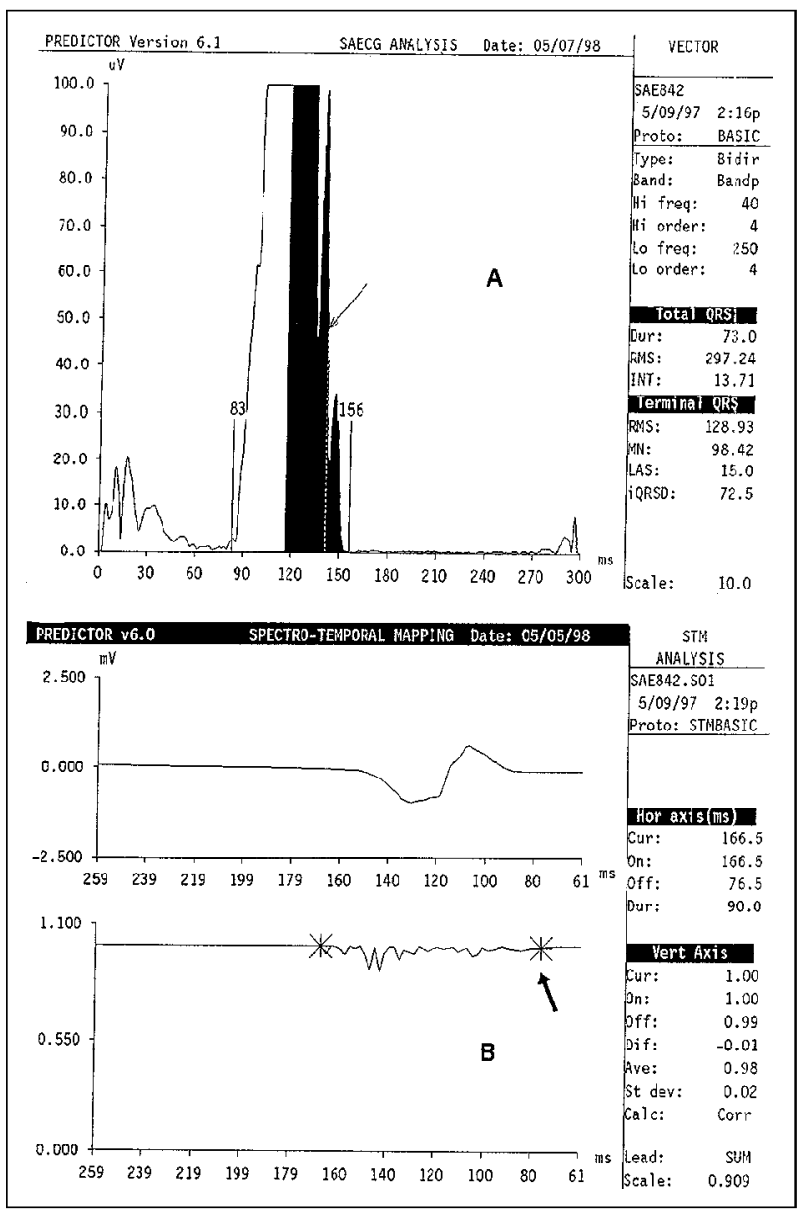

Fig. 3 - ECGAR do caso 10 (CIA). Acima (A), o vetor magnitude do QRS filtrado, de duração normal (73ms). As duas outras variáveis, RMS e LAS também registram valores normais, porém estão invalidadas devido a presença de bloqueio de ramo. Abaixo (B), ECGAR no domínio da freqüência com a análise espectral da correlação intersegmentar linear, durante a inscrição do QRS, da direita para a esquerda (seta), com índices normais de Cem (média = Ave) de 98 e Cedp (desvio-padrão = St dev) de 2 . Linha de base registrando apenas ligeira oscilação, sem evidência de turbulência elétrica ventricular e, portanto, sem potenciais fragmentados.

das paredes do VD, apesar da melhora das condições hemodinâmicas obtidas com a cirurgia.

ECGAR - No DT, das três variáveis analisadas, só a duração total do QRS (DQRS) foi considerada válida, porque correspondia ao aumento no tempo total da ativação ventricular devido ao bloqueio de ramo. A DQRS do grupo

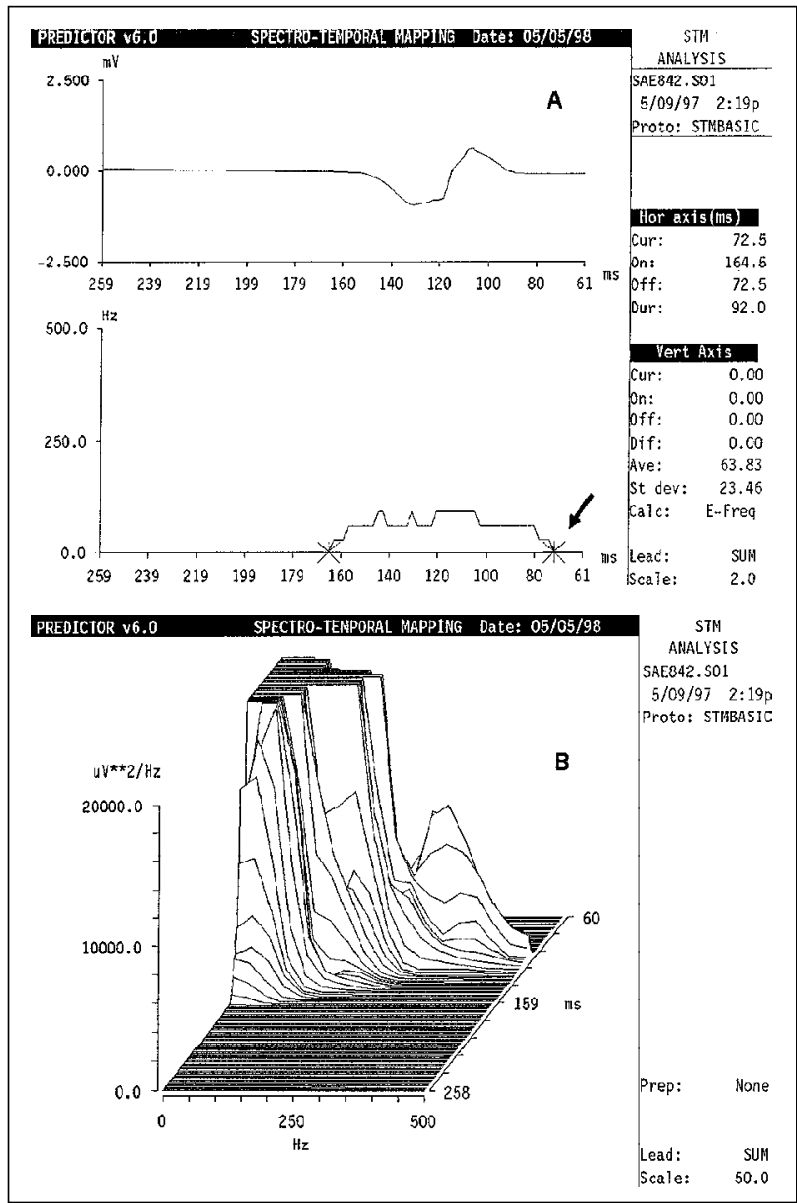

Fig. 4 - Acima (A), ECGAR no domínio da frequiência do caso 10, com a análise espectral, mostrando a banda delimitadora de freqüência de energia do sinal elétrico (seta) com índices de Bdm (média = Ave) de 63,8 e Bddp (desvio-padrão $=$ St dev) de 23,4, considerados normais, sem evidências de turbulência por potenciais fragmentados. Abaixo (B), mapa espectro-temporal do QRS em visão tridimensional, mostrando uniformidade das ondas de frequiência intersegmentar durante a inscrição do QRS, correspondendo a uma imagem espectro-temporal normal.

II foi muito maior que a do grupo I: 137, 1 (ic= 131 a 143,20) vs $87,7(\mathrm{ic}=81,66 \mathrm{a} 93,74)(\mathrm{p}<0,0001)$. As outras duas variáveis, por registrarem eventos apenas nos 40ms finais do QRS, com o alargamento deste pelo bloqueio, tiveram sua análise invalidada, em função do tempo e não foram consideradas (tab. II-fig. 3 e 4$)$. 


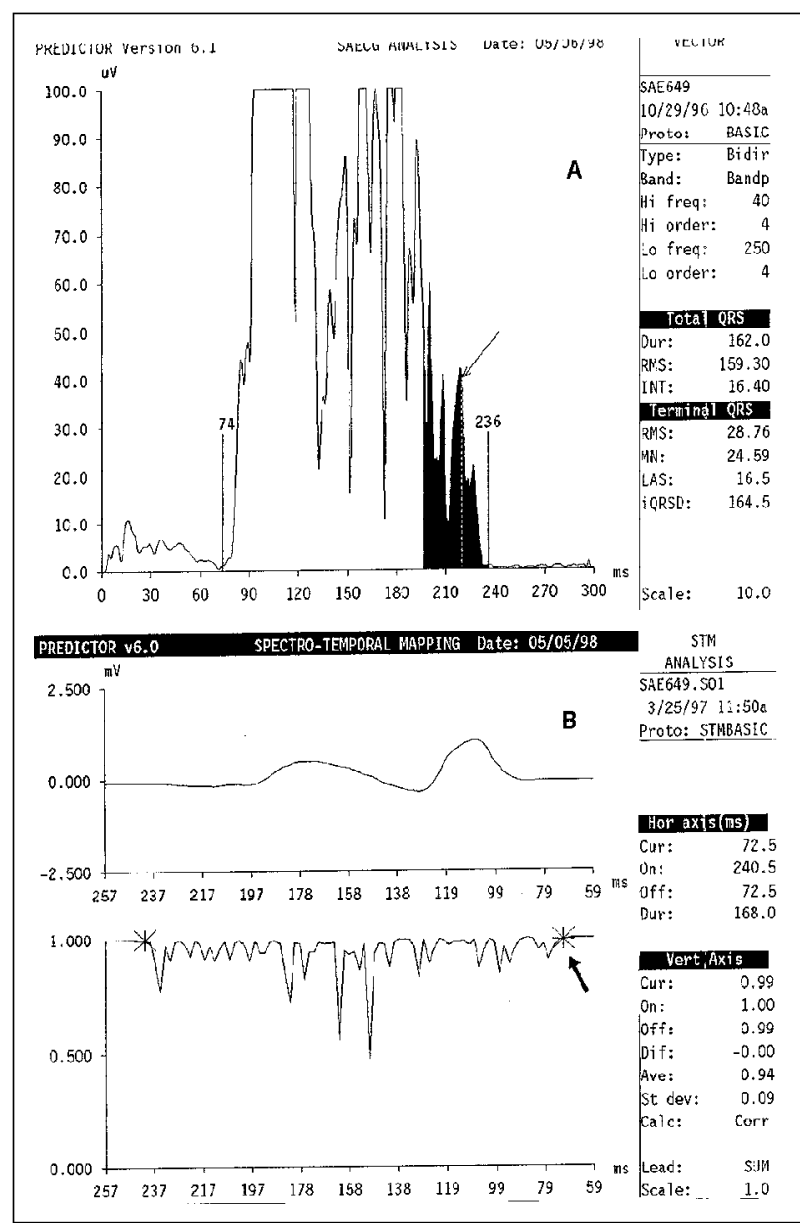

Fig. 5 - ECGAR do caso 3 (tetralogia de Fallot). Acima (A), o vetor magnitude do QRS filtrado, alargado devido ao bloqueio completo do ramo direito, com $162 \mathrm{~ms}$ de duração. Os valores das variáveis RMS $(28,7 \mu \mathrm{V})$ e LAS $(16,5 \mathrm{~ms})$ estão incluídos na área em negro no final do QRS e estão invalidadas. Abaixo (B), o ECGAR no domínio da frequiência com a análise espectral mostrando a correlação intersegmentar linear durante a inscrição do QRS, a partir da direita (seta), com índices anormais de Cem $($ média $=$ Ave $)$ de 94 e Cedp $($ desvio-padrão $=$ St dev $)$ de 9 , correspondentes à presença de turbulência elétrica ventricular (grandes entalhes da linha de base por potenciais fragmentados - comparar com a figura 3 ).

No DF, a Cem do grupo I está dentro dos limites da normalidade (95,3 ic=94,64 a 95,96), enquanto no grupo II é de valor menor (94 ic=93,6 a 94,4,p<0,05); o Cedp tambémé normal no grupo I ( $5,7 \mathrm{ic}=4,99 \mathrm{a} 6,41)$, enquantoé maior no grupo II $(8,1$ ic= 7,26 a 8,94,p p 0,01$)$; e a Bdm no grupo I está dentro dos limites da normalidade $(67,9 \mathrm{ic}=65,56$ a 70,24$)$, enquanto no grupo II está muito mais elevada $(84,4 \mathrm{ic}=81,66$ a 87,14, p<0,0001) (tab. II). O Bddp não teve valores de significância e não foi considerado de interesse (fig. 5 e 6 ).

Considerando os valores destas variáveis e comparando-as com as de crianças normais de faixa etária semelhante, verificamos que no grupo I permaneceram dentro dos limites normais, enquanto no grupo II, a Cem foi menor que o valor normal e a Cedpe Bdm tiveram valores muito mais altos que os normais, ou seja, três dos quatro índices alterados, preenchendo critério para a presença de potenciais tardios com turbulência elétrica durante a inscrição do QRS, apesar da existência do bloqueio de ramo direito (BRD).

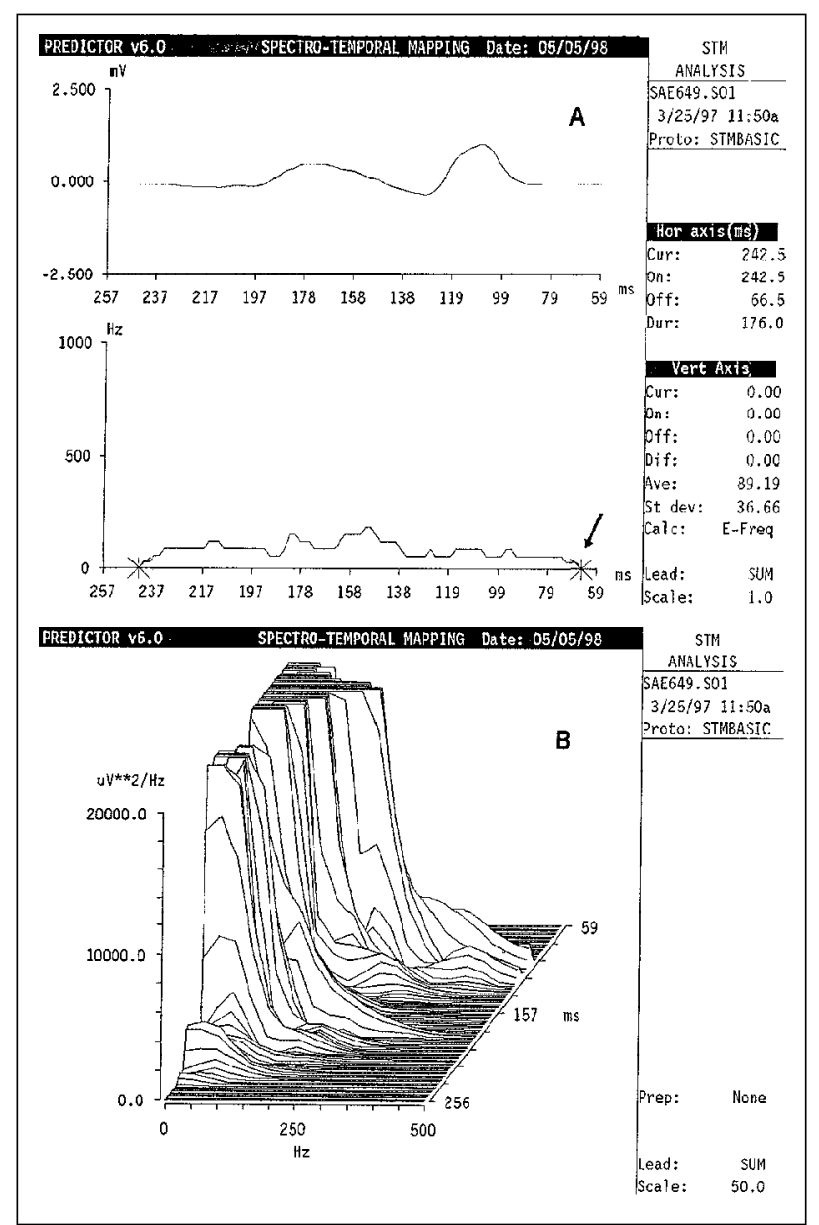

Fig. 6 - Acima (A), ECGAR do caso 3 no domínio da freqüência. Análise espectral mostrando a banda delimitadora de freqüência da energia do sinal elétrico, cujos índices Bdm (média = Ave) de 89,1 e Bddp (desvio-padrão = St dev) de 36,6 (aumentados), correspondem a presença de turbulência elétrica ventricular. Abaixo (B), mapa espectro-temporal tridimensional do QRS, mostrando a grande variação de ondas de frequiência intersegmentares compondo um espectro irregular, devido à turbulência elétrica por presença de potenciais fragmentados ventriculares.

Chamamos a atenção para o fato de que, quando se comparam os valores do grupo I com os de crianças normais de faixa etária semelhante, obtidos em trabalho anterior ${ }^{16}$, verifica-se que no DF, os valores das quatro variáveis do grupo I dos nossos pacientes estão dentro dos limites da normalidade (tab. III), apesar da existência de BRD, ainda que este seja, predominantemente, do tipo incompleto.

\section{Discussão}

OBIRD tem sido causa de grande controvérsia há pelo menos cinco décadas, a partir dos conceitos emitidos por Sodi-Pallares e col, baseados na demonstração de uma "barreira" septal, como principal causa dos atrasos do processo de ativação ventricular nos bloqueios de ramo ${ }^{20,21}$. Para aqueles autores, no caso do BIRD, haveria um ligeiro assincronismo entre a ativação dos dois ventrículos, devido ao atraso sofrido no ramo direito, o que explicaria as configurações rSR' ou RSR' de $\mathrm{V}_{1} \mathrm{e}$ a onda s espessa de $\mathrm{V}_{6}$. 


\begin{tabular}{|c|c|c|c|c|c|c|c|c|c|c|c|c|c|c|}
\hline & pela III - & alores n & e interva & os de cor & $\begin{array}{l}\text { a de cr } \\
\text { s norm }\end{array}$ & $\begin{array}{l}\text { cas com } \\
\text { de faix }\end{array}$ & $\begin{array}{l}\text { aicaçãe } \\
\text { aseme }\end{array}$ & $\begin{array}{l}\text { nteratria } \\
\text { ante }\end{array}$ & IA) & rupo & ompar: & dos ec & s de & \\
\hline \multirow{4}{*}{$\begin{array}{l}\text { Grupo I } \\
\text { CIA } \\
\text { Idade média } \\
5,4 \text { anos }\end{array}$} & \multicolumn{6}{|c|}{ Domínio do tempo } & \multicolumn{8}{|c|}{ Domínio da frequêencia } \\
\hline & \multicolumn{2}{|l|}{$\begin{array}{l}\text { DQRS } \\
\text { Ms }\end{array}$} & \multicolumn{2}{|l|}{$\begin{array}{l}\text { RMS } \\
\mu \mathrm{v}\end{array}$} & \multicolumn{2}{|l|}{$\begin{array}{l}\text { LAS } \\
\mathrm{ms}\end{array}$} & \multicolumn{2}{|l|}{ Cem } & \multicolumn{2}{|c|}{ Cedp } & \multicolumn{2}{|l|}{ Bdm } & \multicolumn{2}{|l|}{ Bddp } \\
\hline & 87,7 & 81,66 & 103,9 & 69,95 & 18,9 & 15,33 & 95,3 & 94,64 & 5,7 & 4,99 & 67,9 & 65,56 & 26,7 & 24,85 \\
\hline & & 93,74 & & 137,85 & & 22,47 & & 95,96 & & 6,41 & & 70,24 & & 28,55 \\
\hline \multirow{3}{*}{$\begin{array}{l}\text { Grupo II } \\
\text { Normais } \\
\text { Idade média } \\
7,5 \text { anos }\end{array}$} & 73,3 & 70,06 & 215,87 & 177,88 & 15,3 & 11,30 & 96 & 95,69 & 5,0 & 4,69 & 64,7 & 63,14 & 24,0 & 22,8 \\
\hline & & 76,54 & & 253,86 & & 19,30 & & 96,31 & & 5,31 & & 66,26 & & 25,12 \\
\hline & $\mathrm{p}=0,002$ & & $\mathrm{p}=0,007$ & & $\mathrm{p}<0,0$ & & $\mathrm{p}=0,5$ & & $\mathrm{p}=0$ & & & $\mathrm{p}=0,09$ & & $\mathrm{p}=0,06$ \\
\hline
\end{tabular}

Observar que no domínio do tempo, as três variáveis estão alteradas no grupo I devido ao bloqueio de ramo direito. No domínio da frequiência, os valores estão nos limites da normalidade ${ }^{16}$.

A controvérsia se estendeu com as observações de Lénègre $^{22}$, que encontrou hipertrofia ventricular direita, histologicamente comprovada, em pacientes que foram à necropsiae que tinham noECG configuração de BIRD ou mesmo bloqueio completo, sem quaisquer lesões no ramo direito.

Outros autores atribuíram as configurações do BIRD não ao bloqueio de ramo propriamente, mas à sobrecarga diastólica do VD na CIA ${ }^{23,24}$ e na $\mathrm{CIV}^{23}$ associada ou não à hipertrofia ventricular direita.

$\mathrm{Na}$ CIA tipo ostium secundum, considerada a forma mais comum, o BIRD é atribuído à sobrecarga de volume diastólico do VD, que existe em cerca de $93 \%$ de crianças com essa malformação ${ }^{18}$.

Mas o padrão de BIRD é observado também em indivíduos sem cardiopatia. Alguns autores relatam uma incidência de cerca de $7 \%$ de crianças normais sem qualquer doença cardíaca ${ }^{25}$, enquanto outros obtiveram configurações de BRD pela simples compressão por cateter da superfície direita do SIV, durante cateterismo cardíaco de indivíduos sem cardiopatia ${ }^{26}$.

Contudo, apesar destas e de outras observações aparentemente discrepantes, parece bem estabelecido o conceito de BIRD, por suas configurações clássicas, de acordo com vários consensos ${ }^{27,28}$, e pela eletrocardiografia contemporânea, como "padrões", "configurações" ou mesmo BIRD.

A nossa casuística esteve constituída de CIA tipo ostium secundum (grupo I) e a incidência de BIRD por critérios de configuração e duração do complexo QRS foi de $100 \%$. Todos os casos apresentaram evidências de crescimento do VD, com a pressão da artéria pulmonar $(\Delta \mathrm{T})$ calculada em torno de $34,5 \mathrm{mmHg}$ (valor médio) que, juntamente com os dados eletrocardiográficos de desvio do eixo elétrico ÂQRS para a direita, $a+120^{\circ}$ e o BIRD, configuram a presença de sobrecarga volumétrica diastólica do VD.

A doença que dá origem ao bloqueio que ocorre no trajeto do ramo, condicionando as configurações clássicas de bloqueio de ramo, pode acometer também a estrutura miocárdica ventricular, como ocorre nos processos isquêmicos, as fibroses das cardiomiopatias, as degenerações gordurosas, as miocardites, os tumores.
A própria sobrecarga ventricular ou a hipertrofia do ventrículo pode converter o subendocárdio em áreas de fibrose derivadas da degeneração de miofibrilas ${ }^{29}$. No VD este fenômeno, que ocorre devido às grandes pressões desta câmara em algumas cardiopatia congênitas, é observado principalmente e, em maior grau, ao nível da implantação da lacínea tricúspide no SIV $^{30}$.

Em nossa casuística, no grupo I, a sobrecarga ventricular direita pôde ser considerada leve ou moderada e de natureza diastólica, não havendo nenhum dado clínico, eletrocardiográfico ou de função hemodinâmica compatível com cardiopatia grave, que pudesse sugerir a presença de alterações estruturais miocárdicas ventriculares. OBIRD de nossos casos é, provavelmente devido às condições hemodinâmicas da CIA, tipoostium secundum, com leve a moderada hipertensão pulmonar, admitindo-se que o miocárdio ventricular direito encontra-se leve ou moderadamente dilatado, sem alterações estruturais de suas miofibrilas.

Já no grupo II, tomado como modelo de BCRD com hipertrofia ventricular direita grave, ocorreram extrasístoles ventriculares isoladas e freqüentes em $29,4 \%$ de casos, acentuado desvio do ÂQRS para a direita a $+168^{\circ}$, e voltagem da R' com 1,9mv, devidos a importante hipertrofia ventricular direita, sendo ainda constatada CIV residual pós-operatória em $35,2 \%$ dos casos, associando-se ainda a esses fatores, a técnica e a manipulação cirúrgicas, todos elementos que poderiam se constituir como causas de alterações estruturais miocárdicas.

No grupo I, não se registrou pelo ECGAR no DF, nenhum caso de turbulência elétrica originada por eventuais potenciais fragmentados ao longo do miocárdio ventricular. A ausência desses potenciais indica que no BIRD, devido a sobrecarga volumétrica do VD, sem alterações da estrutura muscular do VD, não houve turbulência capaz de gerá-la, pelo menos na nossa casuística, em que graus de sobrecarga ventricular direita e de pressão ao nível da artéria pulmonar eram de leve a moderado.

No grupo II, entretanto, todos os casos tinham BCRD com índices do ECGAR no DF anormais, indicando a pre- 
sença de turbulência gerada por potenciais fragmentados, que seguramente foram devidos às condições miocárdicas da tetralogia operada, em que participaram numerosos fatores trans e pós-operatórios. A presença de extra-sístoles ventriculares em quase um terço dos casos é fortemente sugestivo da existência de áreas eletricamente instáveis no VD e, ainda que não tenha ocorrido, até o momento desta comunicação, morte súbita, é necessário estreita vigilância desses pacientes em relação a este evento.

Em conclusão, o BIRD, ele próprio, não foi capaz de gerar potenciais fragmentados ou tardios e turbulência elétrica ventricular, mesmo associado a sobrecarga volumétrica leve ou moderada da CIA, tipo ostium secundum, não associada a outras doenças que deixam seqüelas no miocárdio ventricular.

A ausência de turbulência espectral sugere que, mesmo havendo vias alternativas de condução neste tipo de bloqueio incompleto de ramo, a propagação do estímulo através delas não deu origem à turbulência elétrica. Deste modo, a CIA, tipo ostium secundum, não constituiria uma entidade de risco para desenvolver taquicardia ventricular monomórfica sustentada, ao contrário de alguns casos de TF operada que têm todas as condições para desenvolvê-la, como referido na literatura. Todos estes aspectos, entretanto, poderão ser mais conhecidos com estudos com maior número de casos do que o contido em nossa comunicação.

\section{Referências}

1. Spach MS - Extracellular potentials related to intracellular action potentials during impulse conduction in anisotropic canine cardiac muscle. Circ Res 1979; 45: 188.

2. Spach MS - The discontinuous nature of propagation in normal canine cardiac muscle: Evidence of recurrent discontinuities of intracellular resistance that affect membrane currents. Circ Res 1981; 48: 39.

3. Spach MS - Normal and abnormal propagation in atrial muscle. The role of anisotropy. In: Toubol P, Waldo AL, eds - Atrial Arrhythmias. Current Concepts and Managements. St Louis: Mosby Year Book, 1990: 53

4. Gardner PI, Ursell PC, Pham TD et al - Experimental chronic ventricular tachycardia. Anatomic and electrophysiologic substracts. In: Josephson ME, Wellens HJJ - Tachycardias. Mechanisms, Diagnosis, Treatment. Philadelphia: Lea \& Febiger, 1984:29.

5. Simson MB, Kanosky M, Falcone RA, Dresden C, Josephson ME - Noninvasive methods of predicting patients at risk of malignant ventricular arrhythmias. In: Josephson ME, Wellens HJJ - Tachycardias: Mechanisms, Diagnosis, Treatment. Philadelphia: Lea \& Febiger, 1984: 507-18.

6. El-Sherif N, Restivo M, Craelius W et al - High resolution electrocardiography. Basic and clinical aspects. In: Hombach V, Hilger HH, Kennedy HL Eletrocardiography and Cardiac Drug Therapy. Dordrecht: Kluwer Academic Publis, 1989: 172-203.

7. Breithardt G, Cain ME, El-Sherif N et al - Standards for analysis of ventricular late potentials using high-resolution or signal-averaged eletrocardiography: A Statement by a Task Force Committee of the European Society of Cardiology, The American Heart Association, and the American College of Cardiology. J Am Coll Cardiol 1991; 17: 999-1006.

8. Rubel P, Couderc J Ph, Morlet D, Faynn J, V, Peyrin F, Toubol P- Spectral analysis of high-resolution ECGs. In: Moss AJ, Stern S - Noninvasive Electrocardiology. Clinical Aspects of holter Monitoring. London: WB Saunders, 1996: 291-314.

9. Lander P, Albert D, Berbari EJ - Principles of frequency - domain analysis. In: E Sherif N, Turitto G - High Resolution Electrocardiography. Mount Kisko: Futura Publish, 1992: 105-28.

10. Cain ME, Ambos HD, Witkowski FX, Sobel BE - Fast-Fourier transform analysis of signal-averaged electrocardiograms for identification of patients prone to sustained ventricular tachycardia. Circulation 1984; 69: 711-20.

11. Cain ME, Ambos HD, Markhan J, Fischer AE, Sobel B - Quantification of differences in frequency content of signal-averaged electrocardiogram between patients with and without sustained ventricular tachycardia. Am J Cardiol, 1985; 55: 1500-505.

12. Kelen GJ, Henkin R, Starr AM, Caref EB, Blomfield D, El-Sherif N - Spectral turbulence analysis of the signal-averaged electrocardiogram and its predictive accuracy for inducible sustained monomorphic ventricular tachycardia. Am J Cardiol 1991; 67: 965- 75.

13. Lindsay BD, Markham J, Schechtman KB, Ambos HD, Cain ME-Identification of patients with sustained ventricular tachycardia by frequency analysis of signalaveraged electrocardiograms despite the presence of bundle branch block. Circulation 1988; 77: 122-30
14. Pederson DN, Schamp DJ, Goldberger JJ et al - Spectral temporal mapping of the signal-averaged electrocardiogram for identification of late potentials in patients with bundle branch block. Circulation 1990; 82(suppl III): 739.

15. IpJ, Winters S, Verdino R, TepperD, PeE, Gomes A-Spectral temporal mapping of signal-averaged ECG predicts ventricular tachycardia induction in patients with bundle branch block and intraventicular conduction delay. Pace 1991; 14: 635

16. Ginefra P, Leite MF, Barbosa EC, Barbosa PR, Christiani LA - Avaliação de potenciais tardios em presença de bloqueio de ramo direito no pós operatório da tetralogia de Fallot pelo eletrocardiograma de alta resolução no dominio da frequência. Reblampa 1997; 10: 226.

17. Liebman J, Plonsey R - Eletrocardiography. In: Moss AJ, Adams FH, Emmanouilides GC - Heart Disease in Infants, Children and Adolescents. $2^{\text {nd }}$ ed. Baltimore: Williams \& Wilkins, 1977: 18-61.

18. Garson A Jr - The Electrocardiogram in Infants and Children. A Systematic Approach. Philadelphia: Lea \& Febiger, 1983: 125.

19. Barbosa EC, Barbosa PRB, Ginefra P, Boghossian SH, Albanesi FM - Uma nova técnica de análise pelo ECG de alta resolução no domínio da frequência para reconhecimento de pacientes sob risco de taquicardia ventricular monomórfica sustentada. Reblampa 1997; 10: 227.

20. Sodi-Pallares D, Thomsen P, Barbato E, Soberon J, Fishleder BL, Estandia A Estudio eletrocardiográfico experimental y clínico de los bloqueos incompletos de rama. Arch Inst Cardiol Mex 1948; 18: 497.

21. Rodriguez MI, Sodi-Pallares D - The mechanism of complete and incomplete bundle branch block. Am Heart J, 1952; 44: 715-46.

22. Lénègre $\mathrm{J}$ - Contribution à L'etude des Blocs de Branche. Paris: J B Bailliere et Fils, 1958.

23. DuShane JW - The electrocardiogram in infants and children with low pressure left-to-right shunts. In: Casels DE, Ziegler RF. Electrocardiography in Infants and Children. New York: Grune \& Stratton, 1966: 131-149.

24. Boineau VP, Moore EN, Paterson DF - Relationship between the ECG, ventricular activation, and the ventricular conduction system in ostium primum atrial septal defect. Circulation 1973; 48: 556-64.

25. Burch GE, De Pasquale NP - Eletrocardiography in the Diagnosis of Congenital Heart Disease. Philadelphia: Lea \& Febiger, 1967; 30-105.

26. Peñaloza D, Gamboa R, Sime F - Experimental right bundle branch block in the normal human heart: Eletrocardiographic, vectorcardiographic and haemodynamic observations. Am J Cardiol 1961; 8: 767-79.

27. Hecht HH, Kossmann CE, Childers RW et al - Atrioventricular and intraventricular conduction. Revised nomenclature and concepts. Symposium. Am J Cardio 1973; 31: 232-54

28. Fisch C, Ryan TJ, Willians SV et al - Clinical competence in Eletrocardiography - A Statement for Physicians from the ACP/ACC/AHA Task Force on Clinical Privileges in Cardiology. J Am Coll Cardiol 1995; 25: 1465-9.

29. Lénégre V, Chevalier H, Jacquot R - Étude histologique de sept cas de block de la branche droite. Arch Mal Coeur et Vaiss 1951; 44: 481.

30. Davies MJ, Anderson RH, Becker AE - The Conduction System of the Heart London: Butterworths, 1983: 292. 